\title{
Uso de embalagens e refrigeração na conservação de atemóia
}

\author{
Packing and refrigeration for atemoya preservation
}

\author{
Ana Veruska Cruz SILVA ${ }^{1 *}$, David Guimarães de ANDRADE², Paula YAGUIU², \\ Marcelo Augusto Gutierrez CARNELOSSI ${ }^{2}$, Evandro Neves MUNIZ ${ }^{1}$, Narendra NARAIN ${ }^{2}$
}

\section{Resumo}

O interesse comercial pela atemóia é crescente no Brasil. Sabe-se que é uma fruta com vida útil bastante limitada. O objetivo do presente trabalho foi avaliar a qualidade pós-colheita de atemóias cv. Gefner submetidas a embalagens e armazenamento refrigerado. O delineamento experimental utilizado foi inteiramente casualizado, em esquema fatorial $3 \times 5$ (três embalagens: controle (sem embalagem); embalados individualmente com filme de PVC; colocados em bandejas de poliestireno envolvidas com filme de PVC em cinco tempos de armazenamento), com três repetições. Avaliou-se a perda de massa fresca; coloração da casca e da polpa; Sólidos Solúveis (SS); Acidez Total Titulável (ATT); vitamina C; pH da polpa e atividade de água por ocasião da colheita, a cada três dias de armazenamento. O uso da atmosfera modificada não influenciou na coloração da casca, mas preservou a luminosidade da polpa e reduziu a perda de massa fresca dos frutos. Os teores de SS e ATT aumentaram durante o armazenamento, e foram maiores nos frutos sem embalagens, assim como o teor de vitamina C. O uso de refrigeração foi eficiente na conservação da atemóias 'Gefner', que, após 15 dias de armazenamento ainda apresentavam qualidade para serem comercializados.

Palavras-chave: Annona cherimola $\times$ A. squamosa; atmosfera modificada; qualidade pós-colheita.

\begin{abstract}
Despite the increasing commercial interest in atemoya in Brazil, this fruit has a very limited shelf-life. The present work intended to evaluate the storage of atemoya cv. Gefner under different packing systems and cold storage periods. A factorial of $3 \times 5$ completely randomized design was used (three packing systems: control (unpacked), individually packed with PVC films, and placed in polyester trays wrapped in PVC film for five different storage periods), with three replicates. Weight loss, skin and pulp color, Soluble Solids (SS), Total Tritable Acidity (TTA), vitamin C, pulp pH, and water activity at harvest were recorded every three days of storage. Modified atmosphere did not influence the skin color, but it preserved the pulp brightness and reduced weight loss of the unpacked fruits. SS and TTA levels increased during the storage of unpacked fruits as did the vitamin C contents. Cold storage was efficient for the atemoya preservation, which presented good appearance after 15 days of storage.
\end{abstract}

Keywords: Annona cherimola $\times$ A. squamosa; modified atmosphere; postharvest quality.

\section{Introdução}

O Nordeste brasileiro oferece condições ideais para o cultivo das mais diversas espécies frutíferas de modo que a fruticultura pode contribuir para o desenvolvimento sócio-econômico da região. Porém, o estudo de técnicas de conservação pós-colheita, apesar de ter evoluído nos últimos anos, ainda é escasso e/ou inexistente para algumas frutíferas.

A família Annonaceae compreende um grande número de gêneros e espécies, sendo a maioria nativa de regiões tropicais e subtropicais. Das várias espécies destinadas ao consumo in natura, os fruticultores são atraídos pela atemóia porque este fruto apresenta, sobre a fruta-do-conde, as vantagens de ser mais saboroso, de apresentar sabor doce ligeiramente acidulado e aromático, de conter menor número de sementes, de apresentar vida pós-colheita mais prolongada e, ainda, de permitir maior produtividade (MOSCA; LIMA, 2003).
A introdução da atemóia (Annona cherimola $\times$ A. squamosa) na região Nordeste é recente, havendo uma predominância da cultivar Gefner, de origem israelense, inicialmente cultivada nos projetos de irrigação do Vale do São Francisco. O interesse pela exploração em todo território nacional tem aumentado em razão do lucrativo retorno comercial que a cultura oferece. Isso ocorre devido à possibilidade de se programar diferentes épocas de produção por meio do manejo da poda, irrigação, adubação e polinização, bem como maior adaptação às condições tropicais e subtropicais em relação à cherimóia.

Em geral, a vida pós-colheita das anonáceas é limitada por deterioração fisiológica, causada pelo excessivo amadurecimento do fruto, que exibe rápido amolecimento da polpa e escurecimento da casca, e pelo desenvolvimento de patógenos que ocasionam podridões. Segundo Martinez et al. (1993), por se tratar de um

Recebido para publicação em 15/9/2007

Aceito para publicação em 7/4/2008 (002853)

${ }^{1}$ Embrapa Tabuleiros Costeiros, CEP 49025-040, Aracaju - SE, Brasil, E-mail: anaveruska@cpatc.embrapa.br

${ }^{2}$ Departamento de Engenharia Agronômica, Universidade Federal de Sergipe - UFS, CEP 49100-000, São Cristóvão - SE, Brasil

${ }^{*}$ A quem a correspondência deve ser enviada 
fruto climatérico, as mudanças que causam a perda de firmeza e o escurecimento do fruto devem-se à rápida elevação da taxa de biossíntese de etileno no início do processo de amadurecimento.

O aumento nas taxas da atividade respiratória nas anonáceas, que, segundo Bruinsma e Paull (1984), é induzida pela própria colheita, é seguido de uma rápida modificação na composição química, tornando o sabor e o aroma muito agradáveis. Porém, em contrapartida, há um decréscimo muito rápido da firmeza da polpa (ALVES et al., 1997).

Durante o armazenamento, os frutos acondicionados em filmes plásticos alteram todo o seu metabolismo, devido a estas películas funcionarem como uma barreira para a movimentação do vapor da água, garantindo desta maneira, a manutenção da umidade relativa elevada no interior da embalagem e a turgidez dos produtos. Os filmes plásticos também reduzem sensivelmente a perda de massa dos frutos, retardando o amadurecimento e a elevação das taxas respiratórias, assim como reduzem a produção de etileno e atrasam o amolecimento (perda da firmeza) e várias outras transformações bioquímicas, como a degradação da clorofila e o aparecimento de carotenoides (ZAGORY; KADER, 1988). Porém, segundo os mesmos autores, toda esta mudança na condição de armazenamento do fruto, assim como pode estender a sua vida útil, também pode induzir desordens fisiológicas, caso a permeabilidade da película seja inadequada.

Os filmes plásticos à base de polietileno ou cloreto de polivinila (PVC), devido a sua praticidade, ao custo relativamente baixo e à alta eficiência têm sido bastante utilizados, principalmente quando associados ao armazenamento refrigerado para evitar perdas de frutos (AWAD, 1993; CHITARRA; CHITARRA, 1990). A embalagem com polietileno promove uma modificação na atmosfera ao redor dos frutos devido ao aumento na taxa respiratória, elevando a concentração de $\mathrm{CO}_{2}$ e diminuindo a concentração de $\mathrm{O}_{2}$ (PESIS et al., 1986).

A elevação dos níveis de $\mathrm{CO}_{2}$ e/ou a redução nos níveis de $\mathrm{O}_{2}$ além de retardar o amadurecimento dos frutos (LANA; FINGER, 2000), diminui o metabolismo de pigmentos, de compostos fenólicos e voláteis (BEAURDRY, 1999), reduz a síntese e a ação do etileno sobre o metabolismo dos frutos e a ocorrência de podridões (BRACKMANN; CHITARRA, 1998).

Alguns frutos de alta perecibilidade como o caqui podem ser conservados por dois meses, quando acondicionados em sacos de polietileno com espessura de $0,08 \mathrm{~mm}$ e mantidos a $0{ }^{\circ} \mathrm{C}$ (MARTINS; PEREIRA, 1989). Melo et al. (2002) conseguiram dobrar o tempo de vida útil de cherimóia, passando de duas para quatro semanas quando utilizaram filme plástico zeolite. Com caju, Morais et al. (2002) conseguiram manutenção da qualidade para comercialização até 25 dias de armazenamento, quando os frutos foram envolvidos com PVC.

Assim, a atmosfera modificada pode ser resumida como presença de uma barreira artificial à difusão de gases em torno do produto, aumentando níveis de $\mathrm{CO}_{2}$ e diminuindo os níveis de $\mathrm{O}_{2}$, resultando na alteração na concentração de etileno e vapor d'água e em alterações em outros compostos voláteis (LANA; FINGER, 2000).

No acondicionamento de frutos devem ser aplicadas embalagens ativas, principalmente com absorvedores de etileno, que é um produto indesejável resultante do próprio metabolismo do fruto, funcionando como acelerador de maturação (SARANTOPOULOS et al., 1996).

Além dos cuidados com a embalagem, é necessária atenção na temperatura de armazenamento, para prevenir possíveis danos causados pelo frio. Uma combinação de embalagem com uma temperatura inadequada poderá causar uma entrada de oxigênio e/ou saída de gás carbônico insuficiente através da embalagem, criando uma atmosfera não apropriada ao fruto, podendo provocar desde alterações de cor e textura até o desenvolvimento de sabor e odor não característicos (MILLER et al., 1986), alterações essas que foram verificadas em caqui (ANTONIOLLI et al., 2001). No caso da atemóia, temperaturas muito baixas podem causar injúria pelo frio, alterando a coloração da casca e da polpa, além de provocar outros distúrbios fisiológicos.

$\mathrm{O}$ armazenamento refrigerado associado à utilização da atmosfera modificada destaca-se como uma possibilidade no prolongamento da vida útil dos frutos, refletindo na dilatação do período de comercialização.

O objetivo do presente trabalho foi avaliar a qualidade pós-colheita de atemóia submetida a diferentes embalagens e armazenamento refrigerado.

\section{Material e métodos}

Utilizaram-se frutos de atemóia da cultivar Gefner, oriundos de plantio comercial localizado em Neópolis, Sergipe. A colheita foi realizada na manhã do dia 25 de janeiro de 2005. Em seguida, os frutos foram colocados em caixas plásticas, previamente forradas e transportados ao Laboratório de Tecnologia de Alimentos (LTA), pertencente ao Departamento de Engenharia Química da Universidade Federal de Sergipe (UFS). As atemóias foram mantidas em câmara fria à temperatura de $15^{\circ} \mathrm{C}$ durante todo o experimento.

O delineamento experimental utilizado foi inteiramente casualizado, em esquema fatorial $3 \times 5$, com três repetições. A unidade experimental constou de cinco frutos. Os fatores testados foram embalagens (controle - sem embalagem; frutos embalados individualmente com filme de PVC (espessura e permeabilidade); frutos colocados em bandejas de poliestireno expandido envolvida com filme PVC) e tempo de armazenamento.

As avaliações ocorreram no dia da colheita e a cada três dias de armazenamento para as seguintes características:

a) Perda de massa (\%): os frutos foram pesados após a colheita e a cada dia de avaliação, utilizando uma balança digital GEHAKA, modelo B64400 de 0,1 g de precisão.

b) Cor da casca e da polpa: utilizou-se um colorímetro MINOLTA modelo CR-10. Realizaram-se duas leituras por fruto, sendo uma na casca e outra na polpa. As leituras foram realizadas apontando-se o sensor do aparelho às faces da casca e da polpa do fruto e os parâmetros obtidos formam, 'L', que indica luminosidade (claro/escuro); 'a ', que indica a cromaticidade no eixo da cor verde (-) para vermelha $(+)$; e 'b', que indica a cromaticidade no eixo da cor azul (-) para amarela (+). 
c) Teor de Sólidos Solúveis (SS): utilizou-se um refratômetro manual, modelo WYA ABBE, conforme normas da AOAC (1992), e os conteúdos expressos ${ }^{\circ}$ Brix.

d) Acidez Total Titulável (ATT): foi determinada por titulação, com solução de $\mathrm{NaOH} 0,1 \mathrm{~N}$ e fenolftaleína a $1 \%$ como indicador, e os valores expressos em porcentagem de ácido cítrico.

e) Vitamina C: foi determinada através da técnica de titulação com DCBIB (Diclorofenolendofenol) e os valores expressos em $\mathrm{mg} \times 100^{-1} \mathrm{~g}$ de matéria fresca.

f) $\mathrm{pH}$ da polpa: a leitura foi realizada com um potenciômetro eletrônico, utilizando-se cinco gramas de polpa diluída em $50 \mathrm{~mL}$ de água destilada.

g) Atividade de água $(\mathrm{Aw})$ : foi obtida pela leitura através do determinador de umidade Aqualab (DECAGON) com a colocação de $2 \mathrm{~g}$ de frutos diretamente nas células de análise do aparelho.

\section{Resultados e discussão}

As diferenças significativas na porcentagem de perda de massa entre os tratamentos durante o armazenamento foram destacadas principalmente a partir do sexto dia de armazenamento, continuando até o último dia de avaliação (Tabela 1).

Durante o armazenamento, as maiores perdas de massa ocorrem nos frutos sem embalagem. O comportamento dos frutos em atmosfera modificada passiva (uso da embalagem PVC) mostra que a manutenção da matéria fresca pode ser devido a um possível aumento da umidade relativa do ar no interior da embalagem, saturando a atmosfera ao redor do fruto, o que leva à diminuição do déficit de pressão do vapor de água destes em relação ao ambiente, o que também explica a menor perda de massa nos frutos embalados individualmente. Comportamento semelhante foi observado em atemóia cv. PR3 (YAMASHITA et al., 2002) e em outros frutos, como manga cv. Tommy Atkins (SOUSA et al., 2002), pêssego 'Flordaprience' (KLUGE et al., 1999) e caqui 'Giombo' (ANTONIOLLI et al., 2001).

Tabela 1. Médias de perda de massa fresca (\%) e luminosidade da polpa (L) de atemóia armazenada sob refrigeração em diferentes embalagens.

\begin{tabular}{|c|c|c|c|c|c|c|c|}
\hline \multicolumn{8}{|c|}{ Dias de armazenamento } \\
\hline & 0 & 3 & 6 & 9 & 12 & 15 & Média \\
\hline \multicolumn{8}{|c|}{ Perda de massa fresca (\%) } \\
\hline SE & $0,0^{\mathrm{aE}}$ & $2,9^{\mathrm{aD}}$ & $5,9^{\mathrm{aC}}$ & $8,5^{\mathrm{aB}}$ & $14^{\mathrm{aA}}$ & $15,7^{\mathrm{aA}}$ & $7,8^{\mathrm{a}}$ \\
\hline $\mathrm{BF}$ & $0,0^{\mathrm{aC}}$ & $1,2^{\mathrm{aBC}}$ & $2,1^{\mathrm{BBC}}$ & $2,7^{\mathrm{bAB}}$ & $4,7^{\mathrm{bA}}$ & $4,4^{\mathrm{bA}}$ & $2,5^{\mathrm{b}}$ \\
\hline FI & $0,0^{\mathrm{aC}}$ & $1,2^{\mathrm{aBC}}$ & $1,2^{\mathrm{bBC}}$ & $1,6^{\mathrm{bABC}}$ & $2,4^{\mathrm{cAB}}$ & $3,7^{\mathrm{bA}}$ & $1,7^{\mathrm{c}}$ \\
\hline Média & $0,0^{\mathrm{D}}$ & $1,7^{\mathrm{C}}$ & $3,0^{\mathrm{BC}}$ & $4,3^{\mathrm{B}}$ & $7,0^{\mathrm{A}}$ & $7,9^{\mathrm{A}}$ & \\
\hline \multicolumn{8}{|c|}{ Luminosidade da polpa (L) } \\
\hline SE & $85,3^{\mathrm{aA}}$ & $85,1^{\mathrm{aA}}$ & $80,0^{\mathrm{aAB}}$ & $82,9^{\mathrm{aA}}$ & $76,4^{\mathrm{bB}}$ & $74,4^{\mathrm{bB}}$ & $80,7^{\mathrm{b}}$ \\
\hline $\mathrm{BF}$ & $85,3^{\mathrm{aA}}$ & $84,8^{\mathrm{aA}}$ & $83,9^{\mathrm{aAB}}$ & $83,1^{\mathrm{aABC}}$ & $78,8^{\mathrm{abBC}}$ & $77,7^{\mathrm{abC}}$ & $82,3^{\mathrm{ab}}$ \\
\hline FI & $85,3^{\mathrm{aA}}$ & $84,6^{\mathrm{aA}}$ & $79,8^{\mathrm{aA}}$ & $83,6^{\mathrm{aA}}$ & $82,5^{\mathrm{aA}}$ & $82,3^{\mathrm{aA}}$ & $83,0^{\mathrm{a}}$ \\
\hline Média & $85,3^{\mathrm{a}}$ & $84,83^{\mathrm{a}}$ & $81,2^{\mathrm{bc}}$ & $83,2^{\mathrm{ab}}$ & $79,24^{\mathrm{c}}$ & $78,15^{c}$ & \\
\hline
\end{tabular}

Letras maiúsculas distintas na linha apresentam diferença estatística (DMS-5\%); Letras minúsculas distintas na mesma coluna apresentam diferença estatística (DMS-5\%); $\mathrm{SE}=$ sem embalagem; $\mathrm{BF}$ = bandeja + filme; e FI = filme individual.
Em relação à coloração da casca (Diferencial de Escurecimento - DE), não se observaram diferenças significativas entre os tratamentos, havendo um escurecimento com o decorrer do tempo, que é consequência do processo de amadurecimento dos frutos. Apesar de não haver diferença significativa, os valores encontrados sugerem que os frutos embalados apresentaram uma evolução do DE mais lenta do que os frutos-controle (Figura 1).

Assim como o DE, a Luminosidade (L) também decresceu durante o armazenamento (Tabela 1). Os tratamentos começaram a diferir estatisticamente por volta do sexto dia de armazenamento. No $12^{\circ}$ dia, percebeu-se que os frutos embalados individualmente mantiveram uma maior luminosidade da sua polpa, que também foi observado ao final do experimento ( $15^{\circ}$ dia). Esse comportamento pode ser devido às limitações de trocas gasosas causada pela embalagem, que retarda transformações bioquímicas, como a degradação da clorofila e aparecimento de pigmentos carotenoides responsáveis pelo escurecimento da polpa. É importante relatar que a boa aparência dos frutos foi mantida até o final do armazenamento, não sendo um fator que comprometesse sua comercialização.

Foi observado um aumento no teor de SS, apresentando maiores teores nos frutos sem embalagem, variando de 10 a $31,42^{\circ}$ Brix no $15^{\circ}$ dia de armazenamento (Tabela 2 ). Isso pode ter ocorrido em virtude da perda de água pelos frutos, aumentando a concentração de açúcares por grama de tecido. O expressivo aumento observado nos teores de sólidos solúveis deve-se à transformação das reservas acumuladas durante a formação e o seu desenvolvimento em açúcares solúveis (JERÔNIMO; KANESIRO, 2000). O menor aumento observado no último dia pode estar relacionado com o consumo de carboidratos de reserva dos frutos, para manter a atividade respiratória.

Houve um aumento na ATT com o passar dos dias para todos os tratamentos, sendo que nos frutos sem embalagem essa acidez é mais acentuada (Tabela 2). Diferenças significativas entre os tratamentos foram percebidas a partir do terceiro dia de armazenamento. Os maiores valores de ATT encontrados nos tratamentos sem embalagem devem estar relacionados à

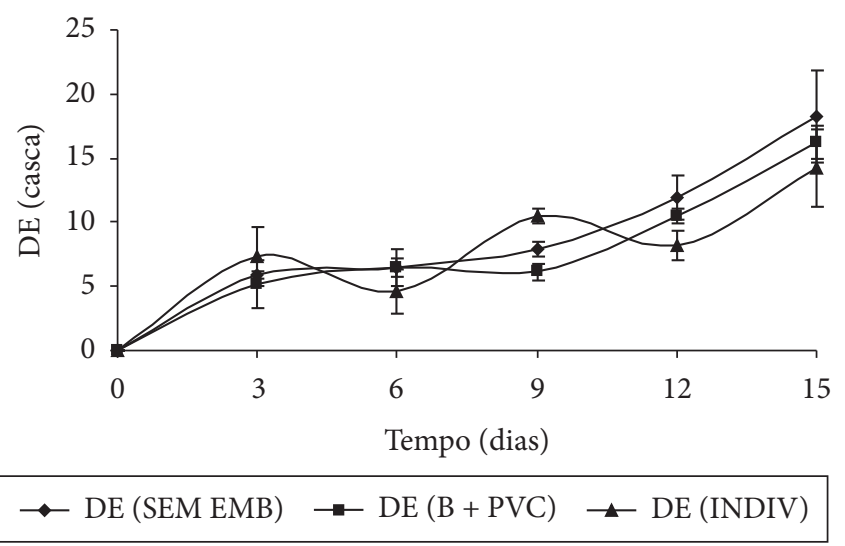

Figura 1. Densidade de escurecimento da casca de frutos de atemóia armazenada sob refrigeração em diferentes embalagens. 
Silva et al.

Tabela 2. Características físico-químicas de atemóia armazenada sob refrigeração em diferentes embalagens.

\begin{tabular}{|c|c|c|c|c|c|c|c|}
\hline \multicolumn{8}{|c|}{ Dias de armazenamento } \\
\hline & 0 & 3 & 6 & 9 & 12 & 15 & Média \\
\hline \multicolumn{8}{|c|}{ SS $\left({ }^{\circ}\right.$ Brix $)$} \\
\hline SE & 10,00 & 15,00 & 22,42 & 27,50 & 31,33 & 31,42 & $22,94^{\mathrm{a}}$ \\
\hline $\mathrm{BF}$ & 10,00 & 14,08 & 19,17 & 26,25 & 28,67 & 29,42 & $21,26^{\mathrm{b}}$ \\
\hline FI & 10,00 & 14,08 & 19,25 & 22,50 & 26,58 & 28,92 & $20,22^{\mathrm{b}}$ \\
\hline Média & $10,00^{\mathrm{e}}$ & $14,39^{\mathrm{d}}$ & $20,28^{\mathrm{c}}$ & $25,42^{\mathrm{b}}$ & $28,86^{\mathrm{a}}$ & $29,92^{\mathrm{a}}$ & \\
\hline \multicolumn{8}{|c|}{ ATT (\% de ácido cítrico) } \\
\hline SE & $0,128^{\mathrm{aC}}$ & $0,128^{\mathrm{bC}}$ & $0,277^{\mathrm{aB}}$ & $0,298^{\mathrm{aAB}}$ & $0,426^{\mathrm{aA}}$ & $0,319^{\mathrm{aAB}}$ & $0,263^{\mathrm{a}}$ \\
\hline $\mathrm{BF}$ & $0,128^{\mathrm{aAB}}$ & $0,085^{\mathrm{aB}}$ & $0,170^{\mathrm{aAB}}$ & $0,234^{\mathrm{abA}}$ & $0,234^{\mathrm{bA}}$ & $0,213^{\mathrm{abAB}}$ & $0,177^{\mathrm{b}}$ \\
\hline FI & $0,128^{\mathrm{aA}}$ & $0,085^{\mathrm{aA}}$ & $0,170^{\mathrm{aA}}$ & $0,149^{\mathrm{bA}}$ & $0,192^{\mathrm{bA}}$ & $0,192^{\mathrm{bA}}$ & $0,153^{\mathrm{b}}$ \\
\hline Média & $0,128^{c}$ & $0,099^{c}$ & $0,206^{\mathrm{b}}$ & $0,227^{\mathrm{ab}}$ & $0,284^{\mathrm{a}}$ & $0,241^{\mathrm{ab}}$ & \\
\hline \multicolumn{8}{|c|}{ Vitamina C (mg.100 $\left.0^{-1} \mathrm{~g} \mathrm{MF}\right)$} \\
\hline SE & $88,88^{\mathrm{aA}}$ & $66,66^{\mathrm{aA}}$ & $74,07^{\mathrm{aA}}$ & $96,29^{\mathrm{aA}}$ & $81,48^{\mathrm{aA}}$ & $59,25^{\mathrm{aA}}$ & $77,77^{\mathrm{a}}$ \\
\hline $\mathrm{BF}$ & $88,88^{\mathrm{aA}}$ & $66,66^{\mathrm{aAB}}$ & $74,07^{\mathrm{aAB}}$ & $37,03^{\text {bв }}$ & $37,03^{\mathrm{bB}}$ & $51,85^{\mathrm{aAB}}$ & $59,25^{\mathrm{b}}$ \\
\hline FI & $88,88^{\mathrm{aA}}$ & $74,07^{\mathrm{aAB}}$ & $74,07^{\mathrm{AAB}}$ & $37,03^{\mathrm{bBC}}$ & $29,62^{\mathrm{bC}}$ & $44,44^{\mathrm{aBC}}$ & $58,02^{\mathrm{b}}$ \\
\hline Média & $88,88^{\mathrm{aA}}$ & $69,13^{\mathrm{abc}}$ & $74,07^{\mathrm{ab}}$ & $56,79^{\mathrm{bc}}$ & $49,38^{c}$ & $51,85^{\mathrm{bc}}$ & \\
\hline \multicolumn{8}{|c|}{$\mathrm{pH}$} \\
\hline SE & $5,58^{\mathrm{aA}}$ & $5,02^{\mathrm{aAB}}$ & $4,31^{\mathrm{bBC}}$ & $4,24^{\mathrm{bBC}}$ & $3,90^{\mathrm{bC}}$ & $4,11^{\mathrm{bBC}}$ & $4,53^{\mathrm{c}}$ \\
\hline BF & $5,58^{\mathrm{aA}}$ & $5,06^{\mathrm{aABC}}$ & $5,50^{\mathrm{aAB}}$ & $4,62^{\mathrm{bBC}}$ & $4,47^{\mathrm{bC}}$ & $5,06^{\mathrm{aABC}}$ & $5,05^{\mathrm{b}}$ \\
\hline FI & $5,58^{\mathrm{aA}}$ & $5,32^{\mathrm{aA}}$ & $5,41^{\mathrm{aA}}$ & $5,41^{\mathrm{aA}}$ & $5,34^{\mathrm{aA}}$ & $5,14^{\mathrm{aA}}$ & $5,36^{\mathrm{a}}$ \\
\hline Média & $5,58^{\mathrm{a}}$ & $5,13^{\mathrm{ab}}$ & $5,07^{\mathrm{abc}}$ & $4,75^{\mathrm{bc}}$ & $4,57^{\mathrm{c}}$ & $4,77^{\mathrm{bc}}$ & \\
\hline \multicolumn{8}{|c|}{ Atividade da água (Aw) } \\
\hline SE & 0,998 & 0,967 & 0,969 & 0,956 & 0,954 & 0,963 & $0,968^{\mathrm{a}}$ \\
\hline $\mathrm{BF}$ & 0,998 & 0,975 & 0,976 & 0,968 & 0,951 & 0,939 & $0,968^{\mathrm{a}}$ \\
\hline FI & 0,998 & 0,970 & 0,974 & 0,975 & 0,953 & 0,961 & $0,972^{\mathrm{a}}$ \\
\hline Média & $0,998^{\mathrm{A}}$ & $0,970^{\mathrm{b}}$ & $0,973^{\mathrm{b}}$ & $0,966^{b c}$ & $0,953^{c}$ & $0,954^{\mathrm{c}}$ & \\
\hline
\end{tabular}

Letras maiúsculas distintas na linha apresentam diferença estatística (DMS-5\%); letras minúsculas distintas na mesma coluna apresentam diferença estatística (DMS-5\%); SE = sem embalagem; $\mathrm{BF}=$ bandeja + filme; e FI = filme individual.

maior perda de água pelos frutos, resultando em uma maior concentração dos ácidos orgânicos presentes no suco celular. Weichman (1986) mencionou que os ácidos orgânicos tendem a diminuir e não a aumentar, durante o amadurecimento dos frutos, em virtude de sua utilização como substrato para a respiração. Essa afirmativa ratifica que o aumento da ATT deve ser consequência, não da síntese de ácidos orgânicos, mas do efeito da concentração em razão da perda de água pelos frutos. Estes resultados também foram encontrados por Yamashita et al. (2002), demonstrando que esse aumento na acidez ao longo do tempo é uma característica peculiar da espécie, o que sugere a este fruto ser conhecido como "pinha com sabor ácido".

Para o teor de vitamina $\mathrm{C}$ foram verificadas diferenças significativas entre os tratamentos, sendo encontrados teores mais elevados dessa vitamina nos frutos sem embalagem (Tabela 2). Isto se deve à grande perda de água verificada nos frutos, resultando em maior concentração em seus tecidos. Em relação ao tempo de armazenamento, a partir do nono dia, iniciou-se uma diminuição dos teores de vitamina C. Vários autores observaram esse comportamento em diversas frutas e hortaliças (LEE; KADER, 2000). Como a vitamina C atua como antioxidante (SMIRNOFF, 1995), sugere-se que esse aumento pode estar relacionado à atuação da vitamina $\mathrm{C}$ como antioxidante, em resposta ao avanço das reações oxidativas que ocorrem durante o armazenamento. Por outro lado, a diminuição dos teores de vitamina $\mathrm{C}$ observada a partir do $12^{\circ}$ dia pode estar relacionada ao avanço do processo de amadurecimento e, por isso, a vitamina $\mathrm{C}$ pode ser considerada um indicador da perda de qualidade dos frutos (KLEIN, 1987). A determinação do conteúdo de vitamina $\mathrm{C}$ é importante, pois, sendo a vitamina mais termolábil, sua presença no alimento indica que provavelmente os demais nutrientes também estão sendo conservados.

Em relação ao pH da polpa, observou-se que os frutos embalados individualmente com PVC mantiveram um comportamento uniforme do $\mathrm{pH}$ ao longo do período de armazenamento, não diferindo estatisticamente em relação ao tempo, enquanto que os não embalados e em bandejas apresentam algumas oscilações (Tabela 2). A manutenção da uniformidade nos frutos embalados individualmente deve-se ao uso dos filmes de polietileno associado ao menor contato do fruto com a atmosfera, o que garantiu um maior retardamento das transformações bioquímicas responsáveis pela alteração do $\mathrm{pH}$ dos frutos.

Durante o armazenamento, foi verificada uma diminuição da atividade de água (Aw) em todos os tratamentos. Nos frutos sem embalagem essa diminuição foi maior, seguida do tratamento em que se utilizou bandeja e filme PVC. Não foi observada diferença significativa entre as diferentes embalagens. Os menores valores 
de Aw nos frutos tratados sem embalagem se devem ao contato direto destes com o ar atmosférico, ou seja, maior perda de água para o ambiente.

\section{Conclusões}

O uso de embalagem com polietileno não influenciou na coloração da casca, mas preservou a luminosidade da polpa e reduziu significativamente a perda de massa dos frutos.

Os teores de sólidos solúveis e acidez total titulável aumentaram durante o armazenamento e foram maiores nos frutos sem embalagens, bem como o teor de vitamina C.

O uso de refrigeração foi eficiente na conservação da atemóia 'Gefner', que apresentaram, aos 15 dias, qualidade para serem comercializados.

\section{Referências bibliográficas}

ALVES, R. E. et al. Colheita e pós-colheita de anonáceas. In: SÃO JOSÉ, A. R. et al. (Eds.). Anonáceas: produção e mercado (pinha, graviola, atemóia e cherimóia). Vitória da Conquista: UESB, 1997. p. 240-256.

A.O.A.C. Official methods as analysis of the association of official analytical chemists. Washington, 1992.

ANTONIOLLI, L. R. et al. Influência da embalagem de polietileno na remoção da adstringência e na qualidade de caquis (Diospyrus kaki), cv. Giombo, armazenados sob refrigeração. Revista Brasileira de Fruticultura, v. 23, n. 2, p. 293-297, 2001.

AWAD, M. Fisiologia pós-colheita de frutos. São Paulo: Nobel, 1993. $114 \mathrm{p}$.

BEAURDRY, R. M. Effect of $\mathrm{O} 2$ and $\mathrm{CO} 2$ partial pressure on selected phenomena affecting fruit and vegetable quality. Postharvest Biology and Tecnology, v. 15, n. 3, p. 293-303, 1999.

BRACKMANN, A.; CHITARRA, A. B. Atmosfera controlada e atmosfera modificada. In: BORÉM, F. M. (Ed.). Armazenamento e processamento de produtos agrícolas. Lavras: UFLA/SBEA, 1998. p. 133-170.

BRUINSMA, J.; PAULL, R. E. Respiration during postharvest development of soursop fruit, Annona muricata L. Plant Physiology, v. 76, n. 1, p. 131-138, 1984.

CHITARRA, M. I. F.; CHITARRA, A. B. Pós-colheita de frutos e hortaliças: fisiologia e manuseio. Lavras: ESAL/FAEP, 1990. 320 p.

JERÔNIMO, E. M.; KANESIRO, M. A. B. Efeito da associação de armazenamento sob refrigeração e atmosfera modificada na qualidade de mangas 'Palmer'. Revista Brasileira de Fruticultura, v. 22 , n. 2 , p. $237-243,2000$.

KLEIN, B. P. Nutritional consequences of minimal processing of fruits and vegetables. Journal of Food Quality, v. 10, n. 3, p. 179-193, 1987.
KLUGE, R. A. et al. Uso de diferentes embalagens plásticas em pêssegos 'Flordaprince' refrigerados. Scientia Agricola, v. 56, n. 4, p. 843-850, 1999.

LANA, M. M.; FINGER, F. L. Atmosfera modificada e controlada: aplicação na conservação de produtos hortícolas. Brasília: Embrapa Hortaliças, 2000. 34 p.

LEE, S. K.; KADER, A. A. Preharvest and postharvest factors influencing vitamin $\mathrm{C}$ content of horticultural crops. Postharvest Biology and Tecnology, v. 20, n. 3, p. 207-220, 2000.

MARTINÉZ, G. et al. Ethylene byosynthesis and physico-chemical changes during fruit ripening of cherimoya (Annona cherimola Mill.) fruit. Journal of Horticultural Science, v. 68, n. 4, p. 477-483, 1993.

MARTINS, F. P.; PEREIRA, F. M. Cultura do caquizeiro. Jaboticabal: FUNEP, 1989. $71 \mathrm{p}$.

MELO, M. R. et al. Conservação refrigerada de cherimóia embalada em filme plástico com zeolite. Bragantia, v. 61, n. 1, p. 71-76, 2002.

MILLER, W. R. et al. Film wrapping mangos at advancing stages of postharvest ripening. Tropical Science, v. 26, n. 1, p. 9-17, 1986.

MORAIS, A. S. et al. Armazenamento refrigerado sob atmosfera modificada de pedúnculos de cajueiro-anão-precoce dos clones CCP-76, END-183 e END-189. Revista Brasileira de Fruticultura, v. 24, n. 3, p. 647-650, 2002.

MOSCA, J. L.; LIMA, G. P. P. Atividade respiratória de atemóia (Annona cherimola Mill. x Annona squamosa L.) cv. Gefner, durante o amadurecimento. In: INTERAMERICAN SOCIETY FOR TROPICAL HORTICULTURE, 2003, Fortaleza. Proceedings... Fortaleza: ISTH, 2003. p. 109-110.

PESIS, E.; LEVI, A.; BEN-ARIE, R. Deastringency of persimmon fruits by creating a modified atmosphere in polyethylene bags. Journal of Food Science, v. 51, n. 4, p. 1014-1016, 1986.

SARANTOPOULOS, C. I. G. L. et al. Embalagens com atmosfera modificada. Campinas: CETEA; ITAL, 1996. $114 \mathrm{p}$.

SMIRNOFF, N. Environment and plant metabolism. Oxford: BIOS Scientific publishers, $1995.270 \mathrm{p}$.

SOUSA, P. J. et al. Influência do armazenamento refrigerado em associação com atmosfera modificada por filmes plásticos na qualidade de mangas 'Tommy Atkins'. Revista Brasileira de Fruticultura, v. 24, n. 3, p. 665-668, 2002.

ZAGORY, D.; KADER, A. A. Modified atmosphere packaging of fresh produce. Food Technology, v. 42, n. 9, p. 70-74, 1988.

WEICHMAN, J. The effect of controled atmosphere storage on the sensory and nutritional qualities of fruits and vegetables. Horticultural Review, v. 8, p. 101-108, 1986.

YAMASHITA, F. et al. Embalagem individual de mangas cv. Tommy Atkins em filme plástico: Efeito sobre a vida de prateleira. Revista Brasileira de Fruticultura, v. 23, n. 2, p. 288-292, 2001. 